\title{
Absolute versus relative class conceptual behavior in squirrel monkeys (Saimiri sciureus)
}

\author{
ROGER K. THOMAS and THOMAS N. CROSBY \\ University of Georgia, A thens, Georgia 30602
}

\begin{abstract}
A theoretical scheme was suggested which distinguished between absolute and relative class concepts and differentiated class from relational concepts. Four monkeys were trained to respond differentially to green and nongreen objects (absolute class) and four responded differentially to odd and nonodd objects (relative class). All monkeys met stringent criteria of performance on their respective tasks, the green-nongreen group in a median of 600 trials and the odd-nonodd group in a median of 1,600 trials $(p=.014)$. Possible explanations for the performance differences and some implications of the theory for comparative, nonhuman conceptual research were discussed.
\end{abstract}

Kendler and Kendler (1975) suggested that endless debates about the meaning of concept can be avoided by accepting Heath's (1967) stricture that the meaning of concept can be determined only in the context of a theory. The "theory" of conceptual behavior used here begins with the condition that evidence for conceptual behavior should be derived from tests of the concept using new stimuli. Otherwise, the logical possibility exists that the animal responded successfully to specific properties or patterns of stimuli. The theory further distinguishes between class concepts and relational concepts and divides class concepts into those based on the absolute properties of stimuli and those based on the relative properties of stimuli.

While the exact form of the theory used here may be unprecedented, there is ample precedent for the kinds of distinctions involved and for using the logical operations to define the structures of concepts (e.g., Haygood \& Bourne, 1965; Hunt, 1962; Millward, 1971; Neisser \& Weene, 1962). Following Whitehead and Russell's Principia Mathematica, Turner (1967) suggested that four logical operations were sufficient to structure all meaningful statements about the universe. Those operations are negation (complement of affirmation), conjunction, disjunction, and implication (conditional). Referring to the logical operations in the terms used by Haygood and Bourne (1965) and Millward (1971), we define class concepts as those determined by the logical operations affirmation and negation and relational concepts as those determined by the explicitly relational operations of conjunction, inclusive-disjunction, conditional, biconditional, and their respective complements. Following Millward, where A and B

Address requests for reprints to Roger K. Thomas, Department of Psychology, University of Georgia, Athens, Georgia 30602 . represented single values $(\mathrm{A}=$ circle and $\mathrm{B}=$ small $)$ on a dimension (e.g., form, size, color), affirmation was exemplified by "all A are + ," conjunction by "all $A$ and $B$ are + ," inclusive-disjunction by "all A or B are +, " conditional by "if A, then it must be $\mathrm{B}$," and biconditional by "A if and only if B." Millward's Table 20.3 (p. 940) includes these examples, examples of their respective complements, and additional explication of the conceptual rules.

In addition to the distinction between class and relational concepts, we also distinguish between absolute and relative class concepts on the grounds that it is not necessary to compare stimuli in order to recognize an absolute class concept, but it is necessary to compare stimuli in order to recognize a relative class concept. For example, "greeness," the absolute class concept in the present work, can be recognized in a stimulus without reference to other stimulus choices, but "oddity," the relative class concept in the present work, can only be recognized relative to the other stimulus choices.

The logical operations form a hierarchy (omitting their complements here) in which affirmation comprises Level I, conjunction, inclusive-disjunction and conditional comprise Level II, and biconditional comprises Level III. The higher levels are defined in terms of, and thus subsume, the lower levels (Neisser \& Weene, 1962). Unless an experimental design demands the use of a higher level operation, it may be possible to interpret the results in terms of a lower level one. Consider Haygood and Bourne's (1965) example of an inclusive-disjunctive concept: "All patterns which are red or square or both are examples"' (p. 178). A subject might learn to select red stimuli, square stimuli, and red-square stimuli by responding to them as separate, concurrent affirmations. While a human subject's explanation might provide evidence for the use of the inclusive-disjunctive operation, research with nonhumans will require 
experimental designs which preclude the use of separate, concurrent affirmations.

In addition to the hierarchy defined in terms of the logical operations, the hierarchy also reflects an order of empirical difficulty for humans (Neisser \& Weene, 1962). Human subjects found Level III concepts to be most difficult to perform and Level I concepts to be easiest. As a first attempt to establish the relative difficulty of absolute and relative class concepts, the present work compared the abilities of independent groups of squirrel monkeys to perform an absolute class concept (greeness) or a relative class concept (oddity). Both concepts were presented in a design which also appears to demand the monkeys' use of the logical operation, conditional. Specifically, the stimuli were presented either on a white tray or a black tray: "if white, then green" or "if black, then nongreen" were correct for one group, and "if white, then odd" or "if black, then nonodd" were correct for the other group. Presumably the role of the conditionals, which was to test for positive and negative uses of the concept, was equated, and any differences seen between the groups were due to differences in the monkeys' abilities to perform these absolute and relative class concepts.

\section{METHODS}

\section{Subjects}

Eight wildborn, adult male squirrel monkeys (Saimiri sciureus) were used. None of these animals had prior experience on any type of conceptual or complex learning problem, nor had they been tested in any apparatus similar to the Wisconsin General Test Apparatus (WGTA). The monkeys were housed in one of our temperature- $\left(24^{\circ}-27^{\circ} \mathrm{C}\right)$ and humidity- $(50 \%-70 \%)$ controlled colony rooms. Timers controlled light onset at 7:00 a.m. and light offset at 7:00 p.m. local time; all testing was done in the light phase. The monkeys received a diet of Purina Monkey Chow (25\% protein) which was supplemented regularly with fresh fruits and vegetables. Water was continuously available.

\section{Apparatus, General Procedures, and Pretraining}

The monkeys were trained and tested in a modified WGTA fitted with a reversible stimulus tray. The tray was white on one side and black on the other, and each side had three foodwells (2.6 cm in diameter, $0.6 \mathrm{~cm}$ deep, $10.3 \mathrm{~cm}$ apart). The foodwells on the black side were painted white inside to facilitate detection of the reinforcers, black currants. The discriminanda for pretraining, described below, were excluded from use in conceptual training. The stimuli used in conceptual training were 235 plastic or wooden objects. Objects to be designated "green" were determined as follows. We first selected the objects that we considered to be green, then we presented them together in a random array with an equal number of objects judged by us to be nongreen independently to a total of 10 male and female graduate students and faculty members in our department; none reported color vision defects. Reading from a standard set of instructions, we asked these subjects to sort the objects into two categories, those he or she judged to be green and those judged to be nongreen. Only the objects for which at least nine of these judges agreed as to the categories were used. This resulted in pools of 96 green objects and 139 nongreen objects. It may be noted that the squirrel monkey is a protanope but appears to be comparable to humans in its responses to wavelengths shorter than about $600 \mathrm{~nm}$ (DeValois \& Jacobs, 1968).
Testing in the WGTA was done only in the illumination provided by a $25-\mathrm{W}$ bulb mounted in the top-center of the apparatus. The monkey was tested in the same room in which it was housed by moving its home cage to an empty slot on the cage rack which was adjacent to the WGTA; screens prevented the other monkeys in the room from observing the ongoing testing. On a given trial after the stimuli had been set and the monkey's door raised, the tray was advanced slightly but not within the monkey's reach. After a 5 -sec delay, the tray was advanced within reach. Thirty seconds were allowed for a response, then the door was closed and a 1-min intertrial interval ensued during which the stimuli for the next trial were set.

Adaptation. Initially, the monkeys were given two 15 -min adaptation sessions, one with the white tray and one with the black tray displayed. Following this were sessions in which (a) one of the foodwells was baited randomly in the monkey's field of view for a total of 25 currants retrieved on each tray brightness; (b) one of the foodwells was baited randomly in the monkey's view, and while still in his view it was covered with a randomly selected object on each trial for totals of 25 currants from each tray; and (c) the previous step was repeated except that the foodwells were baited and covered behind the closed door.

Pretraining. Four object-quality discrimination problems were administered. Two were intended to have form as the dominant cue, and two were intended to have color as the dominant cue. Although tray background was irrelevant, the first and third problems were administered on the white tray, and the second and fourth problems were administered on the black tray. The specific problems in the order that they were administered were: (a) Color-Responses to a black plastic toy teacup were reinforced, and the other two objects on a given trial were selected randomly from a pool of one blue, one yellow, and one red teacup. The positions of the objects were randomly determined on all object-quality discriminations. (b) Form-Responses to a black wooden block were reinforced, and the other two objects on a given trial were selected randomly from the black, blue, yellow, and red teacups. (c) Form-Responses to a blue cardboard box were reinforced, and the other two objects on a given trial were selected randomly from the four teacups previously described. (d) Color-Responses to a white teacup were reinforced, and the other two objects were selected randomly from the four teacups previously described. Training on each problem was continued at a rate of 25 trials per day until a criterion of $92 \%$ correct $(23 / 25)$ was achieved. The objects used in pretraining were not used in subsequent training.

\section{Oddity-Nonoddity Training}

Four of the eight monkeys were randomly assigned to receive this training. Training began the day following the completion of pretraining. With new oddity problems generated for each trial, responses to the odd object were reinforced when the problems appeared on the white tray and responses to either of the nonodd objects were reinforced when the problems appeared on the black tray. The initial member of a nonodd pair of objects was randomly selected from a pool of 51 pairs of objects. The odd objects were taken from a pool consisting of these 51 pairs plus 37 additional objects. Other than the restrictions that the odd object had to differ on a given trial from the nonodd objects in form, color, and size, the selection of the odd objects was random. The order of black and white tray presentations was randomly determined except for the restriction that each tray color had to appear 25 times in each block of 50 trials. To prevent the monkey's use of the auditory cues which accompany reversals of the tray, a simulated tray reversal was done on the trials when no actual change occurred. Training at a rate of 25 trials per session continued until the criterion of $92 \%$ correct in a single session was achieved. The positions of the objects in relation to the foodwells were randomly determined, unless the monkey failed to meet the $92 \%$ criterion in 1,500 trials. In the event of such failure, the center position was no longer used for the odd stimulus, which was restricted to the left or right foodwell. Some literature 
suggests that two-position oddity may be easier to acquire than three-position oddity. Such a change in procedure would not prevent a conclusive interpretation that the monkey's solution of the problem, if achieved, was conceptual.

\section{Green-Nongreen Training}

Except for the differences in cues, the procedures used in green-nongreen training were basically the same as those used in oddity-nonoddity training. The nongreen objects were chosen from a pool of 139. On a given trial, the two nongreen objects were selected randomly from the pool except for the restrictions that the nongreen objects had to differ from each other in color, form, and size and had to differ from the green object in color, form, and size. Responses to the green object (chosen randomly from a pool of 59) were rewarded on the white tray and responses to either of the nongreen objects were rewarded on the black tray. Following the achievement of $92 \%$ correct in one session of 25 trials, a generalization test was given using a new pool of 37 green objects. The nongreen objects were randomly selected from the old pool of 139 .

\section{RESULTS AND DISCUSSION}

All subjects met criterion $(92 \% ; 23 / 25)$ on all pretraining, object quality discrimination problems, and there were no significant differences between the animals assigned to the absolute and relative class concept tasks. The mean numbers of trials to criterion on the two color problems were 94 and 150; for the two form problems, these values were 69 and 38. All monkeys had significant "runs" ( $p<.001)$ of correct responses during the criterion block of trials on all problems (Grant, 1947).

All subjects met the $92 \%$ criterion on their respective tests of class concepts. To indicate that the $92 \%$ criterion was unlikely to have been reached by chance on the conceptual tasks, a significant "runs" analysis was done as follows. The joint probability of a correct response in a white tray $(p=1 / 3)$ and black tray $(p=2 / 3)$ pair of trials is $2 / 9$. A "run" of correct responses based on units representing an average of such pairs may be conservatively assessed using Grant's (1947) procedures. Grant's tables for $p=1 / 3$ may be used by basing the analysis on half the absolute number of correct responses and half the total number of trials (i.e., by using white-black trial pairs as the relevant unit). Earlier (Thomas \& Kerr, 1976), the significance of criterion performance was assessed by determining the separate probabilities of white tray and black tray "runs" and multiplying these to determine the joint probability of both. The procedure used here may be preferable owing to its being more conservative and to its being less susceptible to the effects of the standard errors associated with the separate "runs"' analysis."

Table 1 shows trials to criterion, percentages correct in the last session, and probabilities associated with "runs" of correct responses for each monkey. The difference in median trials to criterion for the absolute $(600)$ and relative $(1,600)$ class con-
Table 1

Trials to Criterion, Percentage of Correct Responses in Final Session, Longest "Run"/Trials to End of Run in the Final

Two Sessions (see text), and Associated Probabilities that the "Runs" Occurred by Chance for the Absolute and Relative Class Groups

\begin{tabular}{ccccl}
\hline Subject & Trials & \% Correct & "Runs"/Total & \multicolumn{1}{c}{$p<$} \\
& \multicolumn{4}{c}{ Absolute Class Concept } \\
S5 & 600 & 100 & $13 / 300$ & .0002 \\
S6 & 950 & 96 & $8 / 475$ & .05 \\
S7 & 600 & 96 & $12 / 300$ & .0004 \\
S8 & 325 & 96 & $12 / 163$ & .0002 \\
& \multicolumn{4}{c}{ Relative Class Concept } \\
S1 & 1175 & 96 & $11 / 587$ & .002 \\
S2 & 1550 & 100 & $12 / 775$ & .001 \\
S3 & 1750 & 92 & $13 / 875$ & .0004 \\
S4 & 1650 & 96 & $11 / 825$ & .0031 \\
\hline
\end{tabular}

cept groups was statistically significant (Mann Whitney $U=0, p=.014$ ).

Three monkeys were tested for generalization of the absolute class concept using a new pool of 37 green objects. In the first and only 25 -trial session, S6 had a score of $100 \%$ correct with a green run of $13(\mathrm{p}<.001)$ and a nongreen run of $12(\mathrm{p}<.01)$; S7 had $96 \%$ correct with a green run of $9(p<.001)$ and a nongreen run of $13(\mathrm{p}<.009)$; and S8 had $92 \%$ correct with a green run of $8(\mathrm{p}<.001)$ and a nongreen run of $9(\mathrm{p}<.07)$. The generalization test was included as further evidence against the interpretation that the monkeys learned the specific properties of the 59 original green objects. Even without the generalization test, learning specific properties was unlikely, since responses to the green objects were both positively and negatively reinforced depending on the tray on which they were presented. If a monkey learned the specific properties, he also had to learn that they were associated with different reinforcement contingencies in the different contexts provided by tray color.

Figures 1 and 2 show individual acquisition curves for the absolute and relative class concepts, respectively. Trials on the white tray (concept positive) and trials on the black tray (concept negative) were plotted separately, in part to assess whether acquisition of one aspect (positive or negative) differed from that of the other. Given the generally parallel acquisition curves, the tendency of both groups to perform better on the black tray probably reflects nothing more than the differential probabilities of responding correctly by chance $(67 \%$ vs. $33 \%)$. Evidence for differential learning might have been indicated if performance on one aspect reached and sustained, say, $90 \%$ correct significantly prior to performance on the other aspect. Monkey S6 in Figure 1 provided the closest approximation to such a result. 

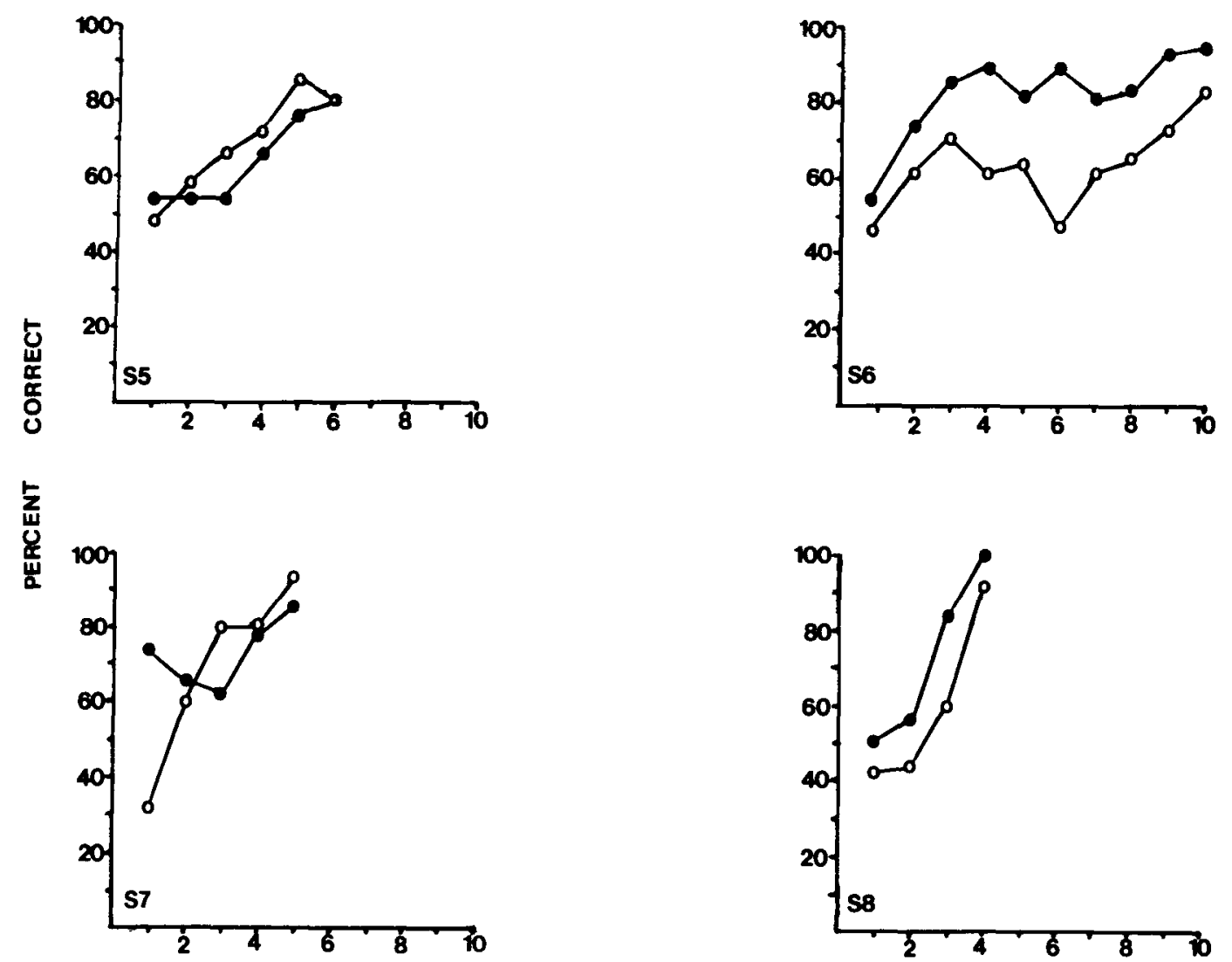

BLOCKS OF 50 TRIALS

Figure 1. Individual acquisition curves plotted separately for green positive on white tray trials and green negative on black tray trials.

It was necessary to use two-position oddity with three monkeys (S2, S3, and S4), as they did not meet the $92 \%$ criterion in 1,500 trials. Other than the subsequent achievement of criterion in 250 trials or less, it is difficult to assess the effects of the change to two-position oddity, since the curves (Figure 2, particularly the plots after Block 15) do not appear to depart appreciably from that of the monkey (S1) which met criterion on three-position oddity. Criterion level performances appear to occur somewhat abruptly in either case, although it should be recognized that this occurs against a background of generally steady improvement. Two-position oddity improves the chances of correct responding owing to the elimination of the center foodwell as a reinforcement possibility, and one may speculate that the Gestalt resulting from the two nonodd and perceptually identical objects being adjacent offers advantages in discrimination.

\section{DISCUSSION}

The results of the present study suggest that, among class concepts, those based on the absolute properties of stimuli may be easier for a squirrel monkey to learn and perhaps to perform than those based on the relative properties of stimuli. However, there may be more difficult absolute class concepts than greenness or easier relative class concepts than oddity, and additional comparisons of absolute and relative class concepts will be needed before a general conclusion about their relative difficulty may be justified.

The learning-performance distinction addressed here is one which asks whether there is an inherent difference between an animal's ability to recognize (and, therefore, perform on the basis of) greenness vs. oddity. One may speculate that greenness and oddity are both inherently recognizable by species which have the requisite visual abilities. Nissen (1953) seemed to have offered this kind of speculation when he suggested, "Response to identity and difference (of stimulus qualities and intensities) seems to be a primitive and universal phenomenon in animal behavior"' (p. 286) and, similarly, Rensch (1967) when he said that the ability to recognize equality and differences in sensations was "an inborne mental function ... a phenomenological fact that cannot be analyzed further"' (p. 49). In any event, whether the abilities to recognize greenness and oddity are in- 


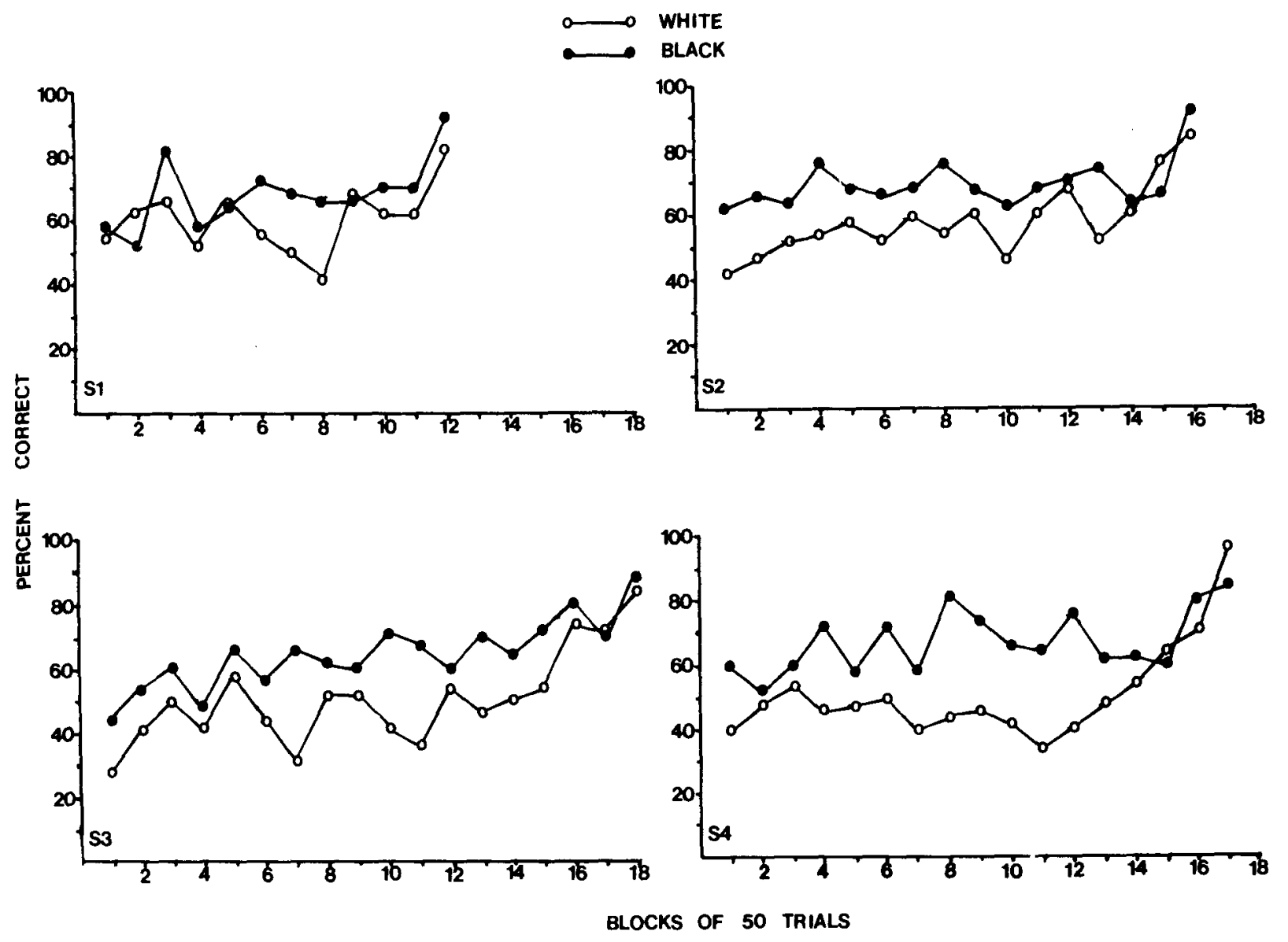

Figure 2. Individual acquisition curves plotted separately for odd positive on white tray and odd negative on black tray trials.

herent and whether there are inherent differences in these abilities may be difficult if not impossible to determine.

On the other hand, there is a basis to suggest that there may be differences in a monkey's opportunities to learn the reinforcement contingencies associated with the greenness vs. the oddity concepts in the present work. As a framework for discussion, Harlow's (1959) error factor theory is useful. Following Harlow's theory, the stimulus objects' positions, form, size, and color were ambiguous with respect to the reinforcement contingencies and might have provided erroneous cues for the odditynonoddity group. However, color was not ambiguous in this respect for the greennessnongreenness group; therefore, this group had fewer cues on which to base erroneous responses. Additional support for error factor theory might be found in the rapid improvement seen with the three monkeys that were shifted after 1,500 trials from three-position to two-position oddity. The partial reduction in position ambiguity which resulted from the elimination of the center position as a location for the odd object might account for the improvement, but the improvement might have been the result of the number of trials which had accrued.

In terms of the present theory and its requirement that tests for conceptual behavior be conducted using new stimuli, with two possible exceptions we are unaware of evidence for conceptual behavior in nonprimate animals which may not be described as involving absolute class concepts. One exception is that some nonprimate species have shown significant improvement on object quality learning sets (see Bitterman, 1975; Warren, 1974), a result which we suggest may require the animal's use of conceptual conditionals. Nevertheless, the improvement shown on object quality learning set tasks by nonprimates has not been particularly impressive. The other exception is that Mueller (1975) has shown that hawks (Falco sparverius) select odd prey. It remains to be seen whether the hawks' conceptual oddity behavior is specific to such biologically significant stimuli. Strong and Hedges (1966) and Thomas and Boyd (1973) discussed the methodological problems which had precluded conclusive demonstrations of conceptual oddity behavior in previous investigations using nonprimate animals. However, the nonprimates' abilities to perform conceptual oddity tasks are not in question at this time, because the 
appropriate oddity tasks have not been used with nonprimate animals.

In view of our suggestion that conclusive evidence for conceptual behavior in nonprimates has been provided only for absolute class concepts (with the two exceptions noted) and in view of the numerous and impressive demonstrations of conceptual behavior in pigeons exemplified in the work of Herrnstein, Loveland, and Cable (1976), it may be useful to justify the former view in the face of the latter. Their pigeons responded appropriately to new photographs representing the concepts "tree," "water," and "a person." According to the present theory, an absolute class concept is one which meets the conditions (a) that the logical operations affirmation and negation are appropriate and (b) that it is not necessary to compare stimuli in order to affirm the stimulus which manifests the concept. The second condition was readily met in Herrnstein et al.'s work, as the trained pigeon need only look at an instance of a concept to affirm it; a photograph of a tree is affirmable as such if one has the concept.

However, that affirmation was the appropriate logical operation may seem questionable in view of Herrnstein et al.'s (1976) description of "trees" as "a complex list of probabilistic conjunctions and disjunctions," although they continued, "the discovery of which would require far more effort than seems justified by any possible benefits"' (p. 298). Furthermore, Herrnstein et al.'s discussion of their inability to identify common elements among the stimuli might appear to question the appropriateness of affirmation in that the stimuli might seem to have nothing in common to be affirmed. However, Herrnstein et al. may not have considered that the elements in their stimuli might have formed affirmable "compounds" with properties which did not depend on the presence of a given element.

The preceding suggestion is not farfetched in view of Rescorla's (1973) work in which he described "a compound stimulus, $\mathrm{AB}, \ldots$ composed of the individual $A$ and $B$ elements as well as a separate stimulus unique to their combination ... [which] ... can acquire associative strength ... and which is attenuated when the unique stimulus becomes irrelevant to reinforcement"' (p. 331). It is suggested here that stimuli comprising concepts such as "tree," "water," and "a person" may have properties which are analogous to those described for Rescorla's "unique stimulus," provided one accepts that compounds such as tree, water, and a person consist of many elements, no one of which is essential to their "unique stimulus" properties. Support for this latter assumption may be found in the work of Morgan, Fitch, Holman, and Lea (1976), who trained pigeons to respond conceptually to various forms (typefaces, handwritten) of the letter " $\mathrm{A}$ " and the number "2." Morgan et al. also trained their pigeons on As and $2 s$ with parts removed and obtained results which led to the conclusion that the pigeons' "performance was controlled by several features, none of which alone could be considered necessary or sufficient"' (p. 57).

In addition to the use of their respective class concepts, the monkeys in the present work were required to use a relational concept, a conditional of the "if, then" form. Again, with the exception of learning set tasks, we are unaware of any conclusive uses of conceptual conditionals by nonprimate animals. There are at least two previous experiments which provided conclusive evidence for the use of conceptual conditionals by monkeys, one from this laboratory using Saimiri sciureus (Thomas \& Kerr, 1976) and one by Riopelle and Copelan (1954) using Macaca mulatta. Except for the obscureness of the first-trial performances on three-trial presentations of new oddity problems, the experiment by Harlow and Moon (1956) might also be regarded as having provided conclusive evidence for conceptual conditional behavior by Macaca mulatta.

Despite the fact that the more convincing demonstrations of conceptual behavior appear to have been limited largely to pigeons and primates, we do not suggest that this reflects the probable scope of such behaviors in the animal kingdom. To the contrary, we suggest that too few conclusive investigations of conceptual behavior in nonhuman animals have been done.

\section{REFERENCES}

Bitterman, M. E. The comparative analysis of learning. Science, 1975, 188, 699-709.

DeValois, R. L., \& Jacobs, G. H. Primate color vision. Science, $1968,162,533-540$.

GRANT, D. A. Additional tables of the probability of "runs" of correct responses in learning and problem solving. Psychological Bulletin, 1947, 44, 276-279.

HaRLow, H. F. Learning set and error factor theory. In S. Koch (Ed.), Psychology: A study of a science, (Vol. 2). New York: McGraw-Hill, 1959.

HaRLow, H. F., \& Moon, L. E. The effects of repeated doses of total-body $\mathrm{X}$ radiation on motivation and learning in rhesus monkeys. Journal of Comparative and Physiological Psychology, 1956, 49, 60-65.

HAygood, R., \& Bourne, L. E. Attribute and rule learning aspects of conceptual behavior. Psychological Review, 1965, 72, 175-195.

HEATH, P. L. Concept. The encyclopedia of philosophy (Vol. 2). New York: The Free Press, 1967. Pp. 177-180.

Herrnstein, R. J., Loveland, D. H., \& Cable, C. Natural concepts in pigeons. Journal of Experimental Psychology: Animal Behavior Processes, 1976, 2, 285-302.

Hunt, E. B. Concept learning: An information processing problem. New York: Wiley, 1962.

Kendler, H. H., \& Kender, T. S. From discrimination learning to cognitive development: A neobehavioristic odyssey. In W. K. Estes (Ed.), Handbook of learning and cognitive processes (Vol. 1). Introduction to concepts and issues. New York: Wiley, 1975. 
Mueller, H. C. Hawks select odd prey. Science, 1975, 188, 953-954.

MrllwaRd, R. B. Theoretical and experimental approaches to human learning. In J. W. Kling and L. A. Riggs (Eds.), Experimental psychology. New York: Holt, Rinehart \& Winston, 1971.

Morgan, M. J., Fitch, M. D., Holman, J. G., \& Lea, S. E. G. Pigeons learn the concept of an 'A.' Perception, 1976, 5, 57-66.

NeISSER, U., \& WEENE, P. Hierarchies in concept attainment. Journal of Experimental Psychology, 1962, 64, 640-645.

Nissen, H. W. Sensory patterning versus central organization. Journal of Psychology, 1953, 36, 271-287.

RENSCH, B. The evolution of brain achievements. Evolutionary Biology, 1967, 1, 26-68.

REsCoRla, R. A. Evidence for "unique stimulus" account of configural conditioning. Journal of Comparative and Physiological Psychology, 1973, 85, 331-338.

Riopelie, A. J., \& Copelan, E. L. Discrimination reversal to a sign. Journal of Experimental Psychology, 1954, 48, 143-145.

Strong, P. N., JR., \& Hedges, M. Comparative studies in simple oddity learning: I. Cats, raccoons, monkeys, and chimpanzees. Psychonomic Science, 1966, 5, 13-14.
Thomas, R. K., \& Boyd, M. G. A comparison of Cebus albifrons, and Saimiri sciureus on oddity performance. Animal Learning \& Behavior, 1973, 1, 151-153.

Thomas, R. K., \& KERR, R. S. Conceptual conditional discrimination in Saimiri sciureus. Animal Learning \& Behavior, 1976, 4 , 333-336.

TURNER, M. B. Philosophy and the science of behavior. New York: Appleton-Century-Crofts, 1967.

WARREN, J. M. Possibly unique characteristics of learning by primates. Journal of Human Evolution, 1974, 3, 445-454.

\section{NOTE}

1. We thank Frank Miele for generating tables for $p=2 / 3$ and for extending Grant's (1947) tables. Copies of the FORTRAN program may be obtained by writing him at the Department of Psychology, University of Georgia, Athens, Georgia 30602.

(Received for publication January 13, 1977; revision accepted April 21, 1977.) 